\title{
Yellow Picosecond Diamond Raman Laser
}

\author{
Jari Nikkinen ${ }^{1}$, Sean Reilly ${ }^{2}$, Vasili Savitski ${ }^{2}$, Antti Härkönen ${ }^{1}$, Alan Kemp ${ }^{2}$, and Mircea Guina ${ }^{1}$ \\ 1. Optoelectronics Research Centre, Tampere University of Technology, Korkeakoulunkatu 3, 33720 Tampere, Finland \\ 2. Institute of Photonics, University of Strathclyde, Department of Physics, Technology and Innovation Centre, 99 George Street, Glasgow \\ G1 1RD, UK
}

Visible pulsed lasers are required for many applications, for example in biomedical imaging [1] and STED microscopy [2]. Such applications often have stringent requirements on the wavelength and pulse duration, which are not directly available from typical solid-state sources. One method of accessing these wavelengths is to use Raman lasers, with diamond being an excellent Raman laser material $[3,4]$ due to its outstanding thermo-optic properties [5].

Here we demonstrate, to the best of our knowledge, the first monolithic diamond Raman laser, converting pump pulses with duration of $86 \mathrm{ps}$ at $532 \mathrm{~nm}$ to $39 \mathrm{ps}$ pulses at $573 \mathrm{~nm}$. With $\sim 400 \mathrm{~mW}$ of average pump power, $150 \mathrm{~mW}$ of $1^{\text {st }}$ Stokes $573 \mathrm{~nm}$ signal was achieved yielding a $37 \%$ conversion efficiency. As a Raman gain material, we used a synthetic single-crystal diamond (Element 6). The diamond was $0.5 \mathrm{~mm}$ thick and its surfaces were coated to form a monolithic plane-plane Raman laser cavity. The input coupler was highly transparent (HT) for the pump wavelength $(532 \mathrm{~nm})$ and highly reflective (HR) for the $1^{\text {st }}$ Stokes $(573 \mathrm{~nm})$. The output coupling was $82 \%$ reflective for $573 \mathrm{~nm}$ and $\mathrm{HR}$ at $532 \mathrm{~nm}$ to double-pass the pump light. Our pump source was a frequencydoubled SESAM Q-switched microchip MOPA producing 86 ps pulses and $\sim 500 \mathrm{~mW}$ of average power at $100 \mathrm{kHz}$ repetition rate.

The $1^{\text {st }}$ Stokes output power versus pump power is shown in Fig. 1 (left). A few milliwatts of $2^{\text {nd }}$ Stokes and a few hundred microwatts of $1^{\text {st }}$ anti-Stokes signals were observed. The pulse duration was measured with a commercial autocorrelator; a typical autocorrelation trace is shown in Fig. 1 (right).
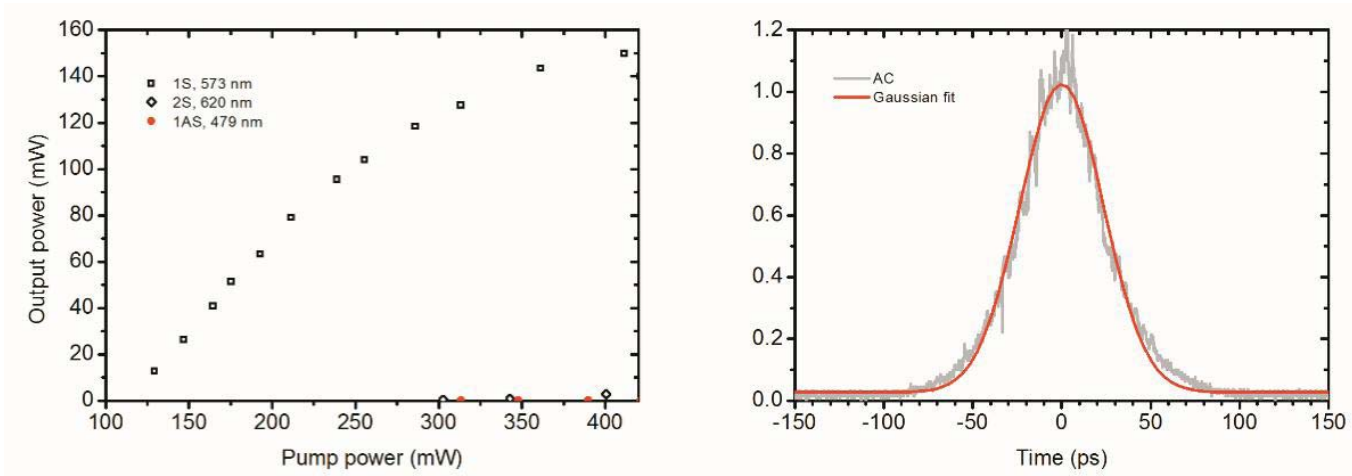

Fig. 1 Left: Output power versus pump power. Right: Typical autocorrelation trace.

In conclusion, we have demonstrated an efficient monolithic Raman diamond laser with conversion efficiency to $1^{\text {st }}$ Stokes reaching $37 \%$. Pulse duration varied with pump power and an average power of $150 \mathrm{~mW}$ was achieved for 39 ps pulses. The system pumped with a 86-ps green Q-switched microchip laser, enables to extend the wavelength coverage to yellow $573 \mathrm{~nm}$ (and potentially to red $620 \mathrm{~nm}$ ) with efficient conversion and significant pulse shortening. An important aspect is the simplicity of the system that enables to reach picosecond visible pulses, previously obtained using mode-locked lasers.

\section{References}

[1] J. Trägårdh, G. Robb, R. Amor, W. B. Amos, J. Dempster, and G. McConnell, "Exploration of the two-photon excitation spectrum of fluorescent dyes at wavelengths below the range of the Ti:Sapphire laser.," J. Microsc. 259(3), 210-218 (2015).

[2] S. W. Hell and J. Wichmann, "Breaking the diffraction resolution limit by stimulated emission: stimulated-emission-depletion fluorescence microscopy," Opt. Lett. 19, 780-782 (1994)

[3] Sean Reilly, Vasili G. Savitski, Hangyu Liu, Erdan Gu, Martin D. Dawson, and Alan J. Kemp, "Monolithic diamond Raman laser," Opt. Lett. 40, 930-933 (2015)

[4] R. Mildren and A. Sabella, "Highly efficient diamond Raman laser," Opt. Lett. 34, 2811-2813 (2009).

[5] I. Friel, S. L. Geoghegan, D. J. Twitchen, and G. A. Scarsbrook, "Development of high quality single crystal diamond for novel laser applications," Proc. SPIE, 7838, 783819-1 (2010). 